\title{
Mitochondrial DNA variability in natural populations of Hawaiian Drosophila. II. Genetic and phylogenetic relationships of natural populations of $D$. silvestris and $D$. heteroneura
}

\author{
Rob DeSalle $\dagger$, \\ L. Val Giddings $\ddagger$ and \\ Ken Y. Kaneshiro*
}

\author{
Department of Biology, Washington University, St. \\ Louis, Missouri, U.S.A.; \\ * Hawaiian Evolutionary Biology Program, Pacific \\ Biomedical Research Center, 3050 Maile Way, \\ Honolulu, Hawaii 96822, U.S.A.
}

Restriction site mapping of mitochondrial DNA with 23 restriction endonucleases was used to examine the genetic and phylogenetic relationships of populations of $D$. silvestris and $D$. heteroneura from the island of Hawaii. Two morphological races of $D$. silvestris are known on the island of Hawaii. One has three bristle rows on the tibia of the foreleg and is found on the east side of the island. The other is found on the west side of the island and has the ancestral bristle row character of two rows on the tibia of the foreleg. All $D$. heteroneura have the ancestral bristle row character state. We demonstrate that mtDNA restriction site analysis can also differentiate the two $D$. silvestris races, and that the two bristle row $D$. silvestris are more closely related to $D$. heteroneura than they are to their three bristel row conspecifics using both distance and character state analysis. Our study (which uses six base recognition restriction endonucleases) is not sensitive enough to determine the phylogenetic relationships of populations within either of the $D$. silvestris lineages.

\section{INTRODUCTION}

Considerable morphological differentiation exists between $D$. silvestris and $D$. heteroneura. They can be distinguished by distinctive body colour differences and pigmentation of the costal margin of the wing and most strikingly by the extreme difference in head shape. This is elongated along the lateral axis in $D$. heteroneura, while the effect is more pronounced in males (Spieth, 1981; Val, 1977; Templeton, 1977). The elongated head is a derived morphological trait as it is absent from $D$. differens and $D$. planitibia, the two closest relatives of $D$. heteroneura and $D$. silvestris which are found on older islands. The distinctive head shape differences are probably secondary sexual characteristics involved in the courtship ritual of these flies (Kaneshiro, 1983). There is considerable ethological isolation between $D$. silvestris and $D$.

†Department of Genetics, Washington University Medical School, St. Louis, Missouri 63110 , $\ddagger$ Office of Technology Assessment, United States Congress, Washington, D.C. heteroneura (Ahearn et al., 1974; Kaneshiro, 1976; Ahearn and Templeton, 1985), although viable and fertile hybrids have been collected from nature (Kaneshiro and Val, 1977) and can be generated in the laboratory (Ahearn et al., 1974; Kaneshiro and Val, 1977; Ahearn and Templeton, 1985).

Attempts to analyse the processes involved in the speciation of $D$. silvestris and $D$. heteroneura have been complicated by the recent discovery that two distinct $D$. silvestris lineages exist and can be identified using morphological (Carson and Bryant, 1979; Carson et al., 1982; Carson, 1983; Carson and Teramoto, 1984; Carson and Lande, 1984) and behavioural data (Kaneshiro and Kurihara, 1982; Kaneshiro, 1983). The presumed ancestral state of two bristle rows is observed in the closely related species $D$. differens (from Molokai) and D. planitibia (from Maui). Populations of $D$. silvestris from the west side of Hawaii as well as all $D$. heteroneura have the ancestral state of two bristle rows. The $D$. silvestris from the east side of Hawaii have a third irregular row of cilia on the dorsal surface of the front tibia. This 
is probably a derived morphological character and is important in the courtship ritual of these flies (Spiess and Carson, 1981; Carson, 1983; Kaneshiro, 1983; Carson and Teramoto, 1984).

Classical approaches to genetic analysis such as studies of chromosome inversions (Carson and Kaneshiro, 1976; Carson, 1982; Carson, 1983) and isozyme analysis (Craddock and Johnson, 1979; Johnson et al., 1975) reveal a high genetic similarity between and within these two species. No fixed isozyme variants nor fixed chromosomal inversions have been found (Carson, 1983). Hunt and Carson (1982) have shown that a small but significant amount of single copy nuclear DNA sequence variation exists ( 0.6 per cent) and we (DeSalle $e t$ al., 1985) have shown the existence of substantial mtDNA restriction site variability. Restriction site analysis of mtDNA may hence be useful in the genetic analysis of these species.

Here we utilise mtDNA variation in a phylogenetic and genetic analysis of $D$. silvestris and $D$. heteroneura to address two major evolutionary questions pertinent to these species. First, what is the genetic relationship between the east side (or three bristle row) $D$. silvestris and the west side (or two bristle row) D. silvestris? Second, what genetic relationships exist between the $D$. silvestris populations and the $D$. heteroneura populations?

\section{MATERIALS AND METHODS}

\section{(i) Flies and DNA}

The individuals examined in this study include the 32 isolines in table 1 of DeSalle et al. (1985). We have included $D$. planitibia of Maui as an outside reference in the phenetic and phylogenetic comparisons of $D$. silvestris and $D$. heteroneura because $D$. planitibia has been implicated as sharing the most recent common ancestor of these two species (Carson and Yoon, 1982; Hunt and Carson, 1982). DNA isolation and manipulation were as described in DeSalle et al (1985). Restriction fragment patterns from an additional seven enzymes to the 16 used in the analysis of DeSalle et al. (1985) were analysed in this study (Bst EII, Bst XI, Nru I, Nco I, Pst I, Sec II and Xba I) to give a total of 23. Complete restriction maps of the individuals analysed can be found in DeSalle (1984) and are available on request.

\section{(ii) Distance measures and phenetic trees}

We have used Ewens et al.'s (1981) distance measure in all phases of this study. Distance measures obtained by the Engels (1981), Nei and $\mathrm{Li}$ (1979) and Hudson (1982) methods are comparable, but not always correlated (Ewens, 1983). Minimal mutational distances were calculated using the phylogenetic analysis using parsimony (PAUP) program, kindly supplied by Dr David Swofford of the Illinois Natural History Survey at the University of Illinois. Unweighted pair grouping with arithmetic averaging (UPGMA) (Sneath and Sokal, 1973) was used to generate phenetic diagrams from the $p$ genetic distance measure of Ewens et al. (1981). PAUP was used to generate Wagner networks from the presence or absence data of each phylogenetically informative restriction site in this study. PAUP was also used to determine the restriction site state of the hypothetical ancestors of closely related individuals.

\section{(iii) Phylogenetic analysis and hypothesis testing}

To determine phylogenetic branching orders we have used PAUP to construct maximum parsimony phylogenies from data on the presence or absence of each restriction site in the various taxonomic units examined. Maximum parsimony phylogenies were then evaluated using Templeton's (1983b) hypothesis testing algorithm. Plausible alternative phylogenies generated on the basis of existing genetic, morphological and biogeographical data were compared to the best mtDNA maximum parsimony phylogenies. The changes in restriction site state required to make a given topology consistent with the data were also computed by PAUP. In this way a list of convergent events for each enzyme and for each topology was obtained. In tests between two topologies, the types of convergence that occur for each restriction enzyme were compared for each topology, and scored according to the criteria of Templeton $(1983 a, b)$. The scores were ranked and the Wilcoxon matched pair test was used to determine the probability that one topology was better than the other. The best maximum parsimony phylogenies and the best alternative phylogenies were examined in light of the two phylogenetic questions posed above.

\section{RESULTS}

\section{(i) Descriptive Results and Phylogeny Estimation}

Table 1 shows the distance matrix of $p$ values for several representative pairwise comparisons of the 
Table 1 Genetic distances for some representative mtDNA haplotypes

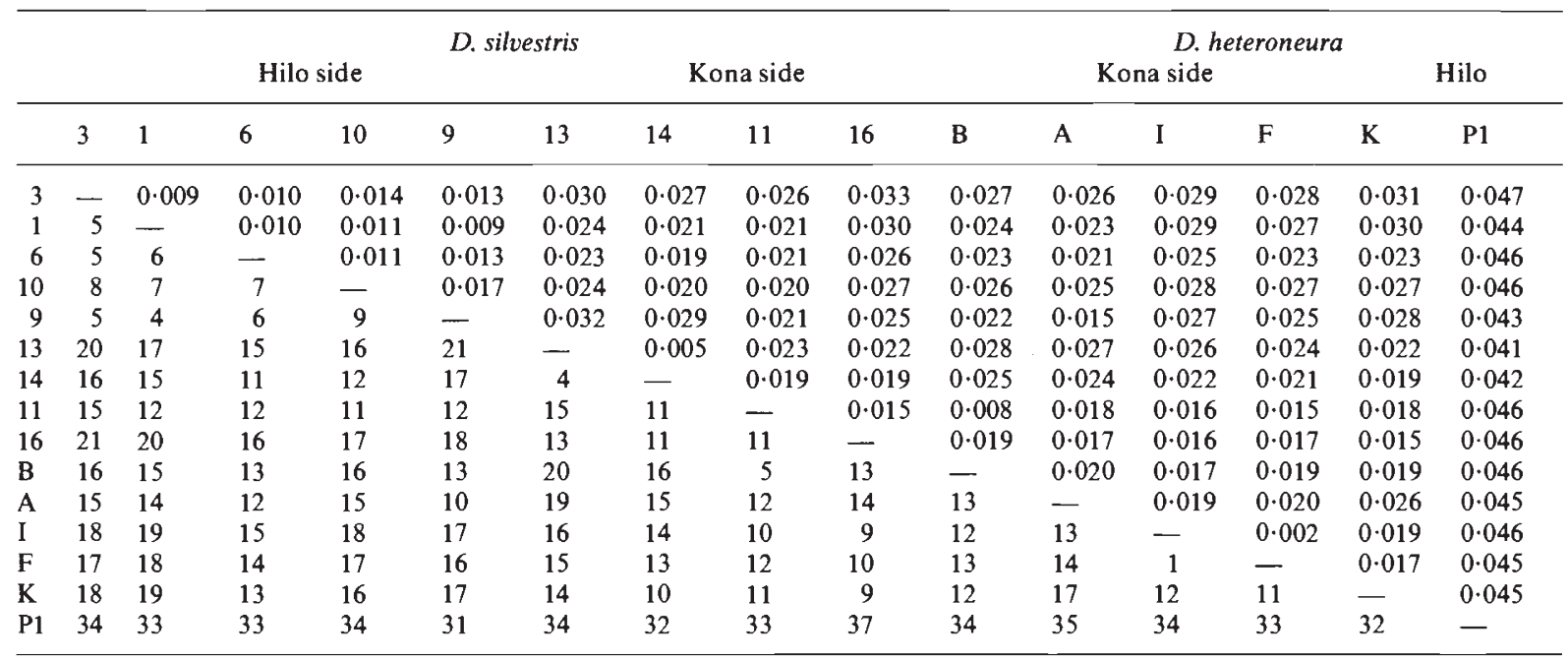

Ewens et al. (1981) $p$ value is given above the diagonal. Minimal mutational distance is given below the diagonal. Isolines are coded as in fig. 2.

28 distinct mtDNA haplotypes. Table 1 also gives the Manhattan distances (minimal mutational distances) for each of these pairwise comparisons. The pairwise distance data for all 32 isolines are summarised in the UPGMA generated phenogram shown in fig. 1 which also shows that some populations, such as the Waihaka $D$. heteroneura, behave as a single cohesive cluster. Other populations, such as the east side $D$. silvestris from Olaa and Piihonua, show a greater degree of heterogeneity. A similar UPGMA analysis of the distance measures from larger population sizes, but for fewer enzymes in DeSalle et al. (1985) reveals the same result. That is, UPGMA analysis of these populations does not reveal a segregation of the Olaa population from the Piihonua population.

Among the five Hualalai $D$. silvestris examined in this study four of the individuals cluster into a closely related set of individuals, but one $D$. silvestris from this locality (W48B2) clusters with the $D$. heteroneura from Hualalai. This observation is discussed in more detail in DeSalle et al. (1985). One unanticipated result of the cluster analysis is that the west side $D$. silvestris cluster with $D$. heteroneura rather than with their east side conspecifics.

Wagner network analysis of presence/absence data is presented in fig. 2. Attempts to find equal minimal length topologies from the data set using Wagner distance methods give the same overall topology as in fig. 2, and changed only the topologies within the major clusters of the network shown. The exact branching orders of the $D$. silves- tris from the east side might be inferred if enough fixed restriction site differences exist. Such an analysis revealed no fixed restriction site differences between the Olaa and Piihonua populations. In fact, these two populations shared five polymorphic restriction sites. The same general trends of relationship, are hence seen in the Wagner analysis as in the UPGMA analysis.

\section{(ii) The use of hypothetical ancestors in estimating phylogeny}

Templeton's (1983b) phylogeny hypothesis testing algorithm often generates nonsignificant comparisons when topologies in which permutations of closely related taxa are tested. Furthermore, the number of possible tree topologies becomes unmanagable when the number of taxa examined is greater than ten (Febsenstein, 1982; Swofford, personal communication). We have therefore attempted to increase statistical power by taking advantage of the fact that extinct intermediates can be inferred from restriction site data using reasoning similar to that employed in constructing phylogenies from chromosomal inversions in Drosophila. This allows us to pool restriction site data from closely related individuals by inferring the hypothetical ancestral state of the mtDNA lineage for each of the localities with more than one such individual.

Analysis of the east side $D$. silvestris with Templeton's algorithm shows that too few informative restriction sites exist to reject any particular 


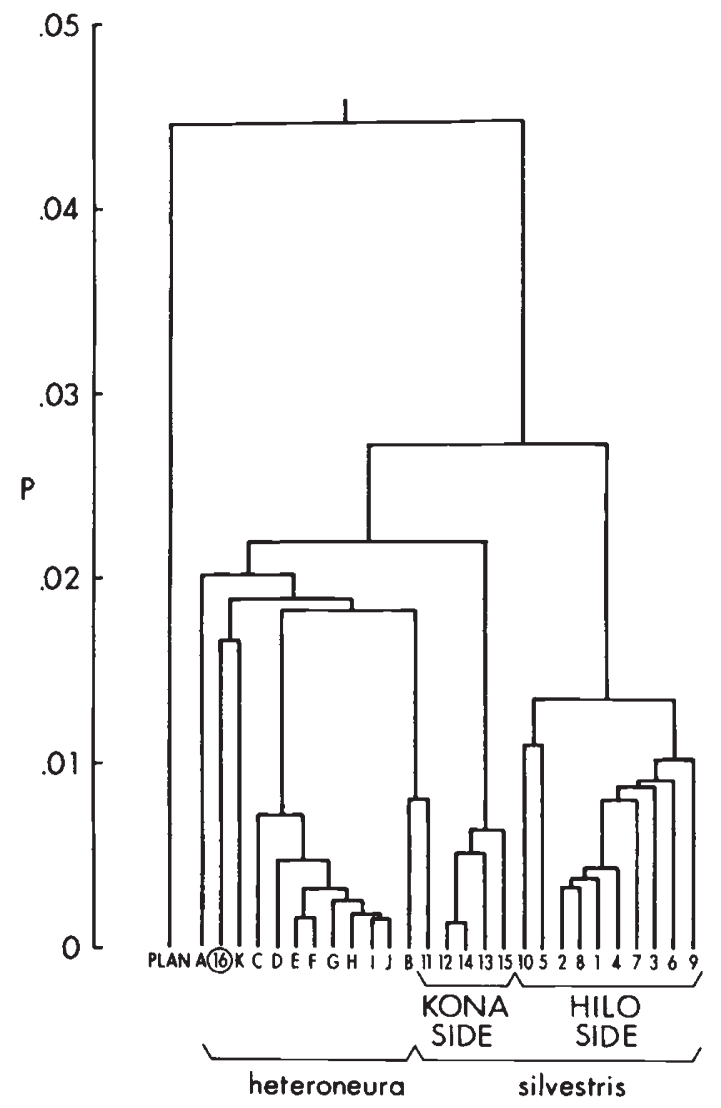

Figure 1 UPGMA generated phenogram of the distance measures for all pairwise comparisons of the isolines examined in this study. $D$. heteroneura isolines are designated by letters (A thru $J$ ) and $D$. silvestris are designed by numbers (1 thru 16). The Kahuku $D$. silvestris is circled. The designations for $D$. silvestris are as follows: $1=\mathrm{U} 28 \mathrm{~T} 2$, $2=$ WPM04, $3=$ WPM04, 4=WPM06, 5=LVGP1, $6=$ LVGP10, $7=$ LVGP12, $8=$ LVGP13, 9=W33B45, $10=$ W12B7, $11=W 48 B 2,12=W 48 G 5,13=W 48 B 1, \quad 14=$ $\mathrm{W} 48 \mathrm{G} 4,15=\mathrm{W} 48 \mathrm{G} 10$, and $16=\mathrm{U} 26 \mathrm{~B} 9$. The designations for $D$. heteroneura are as follows: $A=W 48 G 3, B=W 48 B 6$, $\mathrm{C}=\mathrm{W} 33 \mathrm{~B} 7, \mathrm{~W} 33 \mathrm{~B} 8$ and $\mathrm{W} 33 \mathrm{~B} 13, \mathrm{D}=\mathrm{W} 33 \mathrm{~B} 14, \mathrm{E}=$ W33B5, $\quad F=W 33 B 3, \quad G=W 33 G 10, \quad H=W 33 G 7, \quad I=$ $\mathrm{W} 33 \mathrm{~B} 2, \mathrm{~W} 33 \mathrm{G} 6$ and $\mathrm{W} 33 \mathrm{G} 11, \mathrm{~J}=\mathrm{W} 33 \mathrm{~B} 16$ and $\mathrm{W} 33 \mathrm{~B} 4$ and $\mathrm{K}=\mathrm{Q} 71 \mathrm{G} 12$. Hilo side designates the east side of Hawaii while Kona side designates the west.

branching order. The Maulua isoline, however, appears to be different from other east side $D$. silvestris as shown by UPGMA analysis of distance data. These results suggested that it would be appropriate to determine a hypothetical ancestor for all $D$. silvestris from the east side except for Maulua and to use Maula as a separate taxonomic unit. Similar analysis revealed that it is appropriate to determine the hypothetical ancestors for the Waihaka $D$. heteroneura $(n=13)$ and the Hualalai D. silvestris $(n=4)$. The high degree of similarity of one of the D. silvestris from Hualalai (W48B2) to a Hualalai $D$. heteroneura (W48B6) also suggested that we treat these two as a single mtDNA lineage. Table 2 lists the nine taxonomic units and the states of the phylogenetically informative sites.

Three maximum parsimony phylogenies were generated from the restriction site data on hypothetical ancestors (table 2) with PAUP (fig. 3 ). The common feature of the three phylogenies is the branching of the Hualalai (or two bristle row) $D$. silvestris with $D$. heteroneura. This result suggests that west side $D$. silvestris share a more recent common mtDNA gene pool with $D$. heteroneura than they do with their east side conspecifics. These three phylogenies are all related in that they involve permutations of the branching orders of the west side $D$. heteroneura and the single $D$. silvestris from Kahuku.

Table 2 Restriction site states for the phylogenetically informative restriction sites in the nine mtDNA haplotypes used in PAUP

\begin{tabular}{llllllllll}
\hline & S2 & S8 & H4 & H5 & H7 & S3 & H6 & S1 & P1 \\
\hline a3 & 1 & 0 & 0 & 0 & 0 & 1 & 0 & 1 & 1 \\
a9 & 0 & 1 & 1 & 0 & 0 & 0 & 0 & 0 & 1 \\
b1 & 1 & 0 & 0 & 0 & 0 & 0 & 0 & 1 & 0 \\
f1 & 0 & 0 & 0 & 1 & 1 & 0 & 0 & 0 & 0 \\
f2 & 0 & 1 & 0 & 1 & 1 & 1 & 1 & 0 & 1 \\
g1 & 0 & 1 & 0 & 0 & 1 & 0 & 0 & 0 & 0 \\
g2 & 0 & 0 & 1 & 0 & 0 & 0 & 1 & 1 & 1 \\
g5 & 1 & 0 & 1 & 0 & 0 & 0 & 0 & 1 & 0 \\
g7 & 0 & 0 & 0 & 0 & 1 & 1 & 0 & 0 & 1 \\
j3 & 1 & 0 & 0 & 0 & 1 & 1 & 0 & 1 & 1 \\
h2 & 0 & 1 & 0 & 0 & 0 & 1 & 0 & 0 & 0 \\
h7 & 1 & 1 & 1 & 1 & 1 & 1 & 0 & 1 & 0 \\
h8 & 0 & 1 & 0 & 0 & 1 & 0 & 0 & 0 & 0 \\
m3 & 1 & 1 & 0 & 0 & 0 & 0 & 0 & 1 & 0 \\
m5 & 0 & 1 & 1 & 1 & 1 & 1 & 1 & 0 & 0 \\
k1 & 0 & 1 & 0 & 1 & 1 & 0 & 1 & 0 & 0 \\
k2 & 0 & 0 & 1 & 0 & 0 & 1 & 0 & 0 & 0 \\
o4 & 1 & 0 & 0 & 0 & 0 & 0 & 0 & 1 & 1 \\
p1 & 0 & 0 & 1 & 1 & 0 & 0 & 0 & 1 & 0 \\
s2 & 0 & 0 & 0 & 1 & 0 & 0 & 0 & 1 & 0 \\
r1 & 0 & 1 & 1 & 1 & 0 & 0 & 1 & 0 & 0 \\
t1 & 0 & 1 & 1 & 0 & 1 & 1 & 1 & 0 & 0 \\
u3 & 0 & 1 & 0 & 0 & 1 & 1 & 1 & 0 & 0 \\
\hline & & & & & & & & &
\end{tabular}

$\mathrm{S} 2$ is the $D$. silvestris isoline from Maulua (W12B7); S8 is the $D$. silvestris isoline from Kahuku (U26B9); $\mathrm{H} 4$ is a $D$. heteroneura isoline from Hualalai (W48G3); $\mathrm{H} 5$ is a hypothetical ancestor of $D$. silvestris isoline (W48B2) and $D$. heteroneura isoline (W48B6); $\mathrm{H} 7$ is the $D$. heteroneura isoline from Olaa (Q71G12); S3 is the hypothetical ancestor of the four Hualalai $D$. silvestris isolines (not including W48B2); H6 is the hypothetical ancestor of the thirteen Waihaka $D$. heteroneura isolines; $\mathrm{S} 1$ is the hypothetical ancestor of the nine Hilo side D. silvestris isolines (not including the Maulua isoline W12B7); P1 is the D. planitibia isoline (U84Y)

Restriction sites are as listed in restriction site map appendix of DeSalle (1984) 


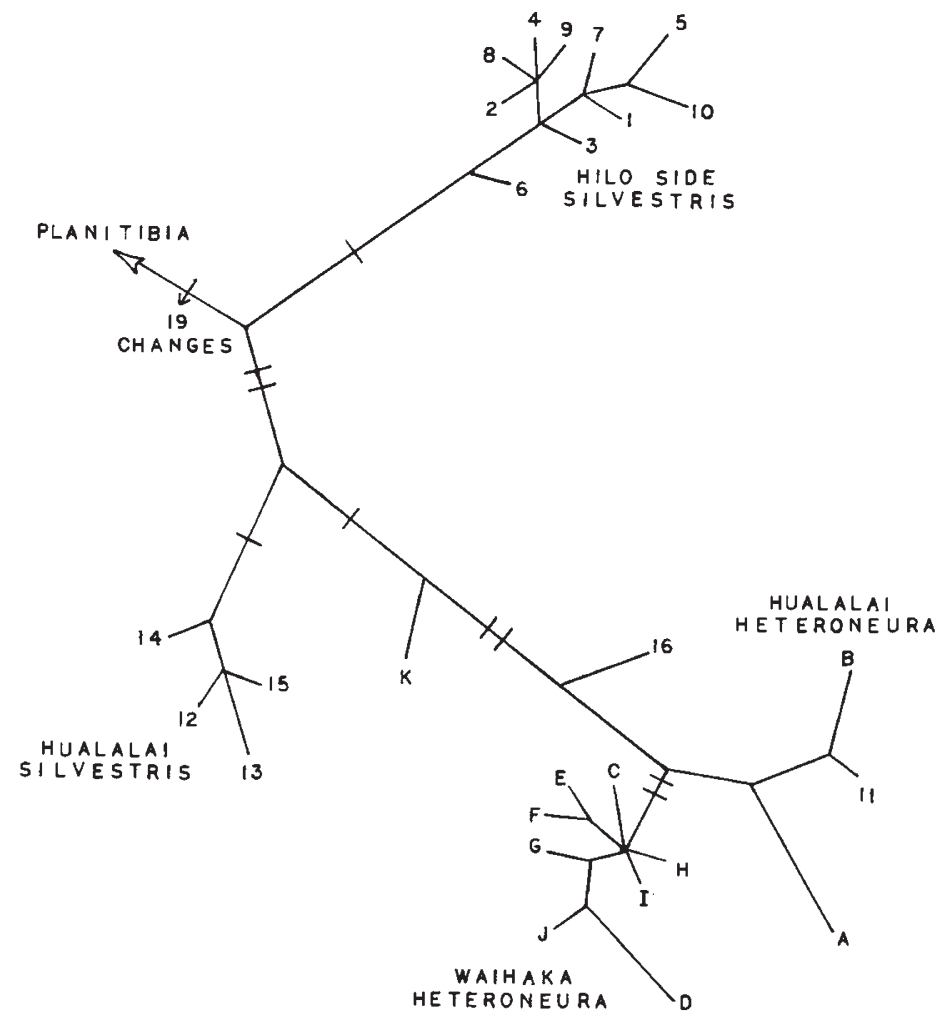

Figure 2 Wagner network for the presence/absence matrix for polymorphic sites. Isolines are coded as in fig. 1. Slashes across branches represent fixed diagnostic restriction sites. Hilo side designates the east side populations.

\section{(iii) Hypothesis testing}

The first major phylogenetic question aderessed is the relationship between the two bristle row and three bristle row $D$. silvestris. In particular, we are interested in whether these two morphologically distinct lineages form two distinct mtDNA lineages. To address this question we compared the phylogenies in fig. 5 using Templeton's algorithm. Phylogeny A represents a topology where the two bristle row $D$. silvestris form a separate branch from the three bristle row $D$. silvestris. Phylogenies B and C represent topologies where the two bristle row $D$. silvestris belong to the same clade or branch as the three bristle row $D$. silvestris. Phylogeny A was significantly better than Phylogeny $\mathrm{B}(p<0.05)$, and nearly significantly better than Phylogeny $\mathrm{C}(p<0.1)$, indicating that the three bristle row mtDNA lineage is indeed distinct from the two bristle row lineage.

In order to expand on the results obtained by maximum parsimony analysis (fig. 3) we tested the three maximum parsimony phylogenies against each other using Templeton's algorithm. Although Phylogeny II is shown to be slightly more probable $(p<0.30)$ than the other two phylogenies (table 3 ), none of them is significantly better than the others. We have chosen to use Phylogeny II in the subsequent comparisons with alternative topologies; however, the same general results are obtained when Phylogenies I and III are used in the comparisons.

We tested the maximum parsimony Phylogeny II against several alternative branching orders, which are proposed from existing behavioural, isozyme, chromosomal inversion, morphological, taxonomic and biogeographic data. One branching

Table 3 Wilcoxon matched pairs signed rank tests for the three maximum parsimony phylogenies in fig. 3

\begin{tabular}{llll}
\hline & I & II vs & I vs \\
& III & III \\
\hline $\begin{array}{l}\text { Number of nonzero ranks } \\
\text { Sum of nonzero ranks }\end{array}$ & $1.5-$ & 2 & 1 \\
Probability & $p<0.30$ & $p<0.25$ & $p<0.50$ \\
\hline
\end{tabular}



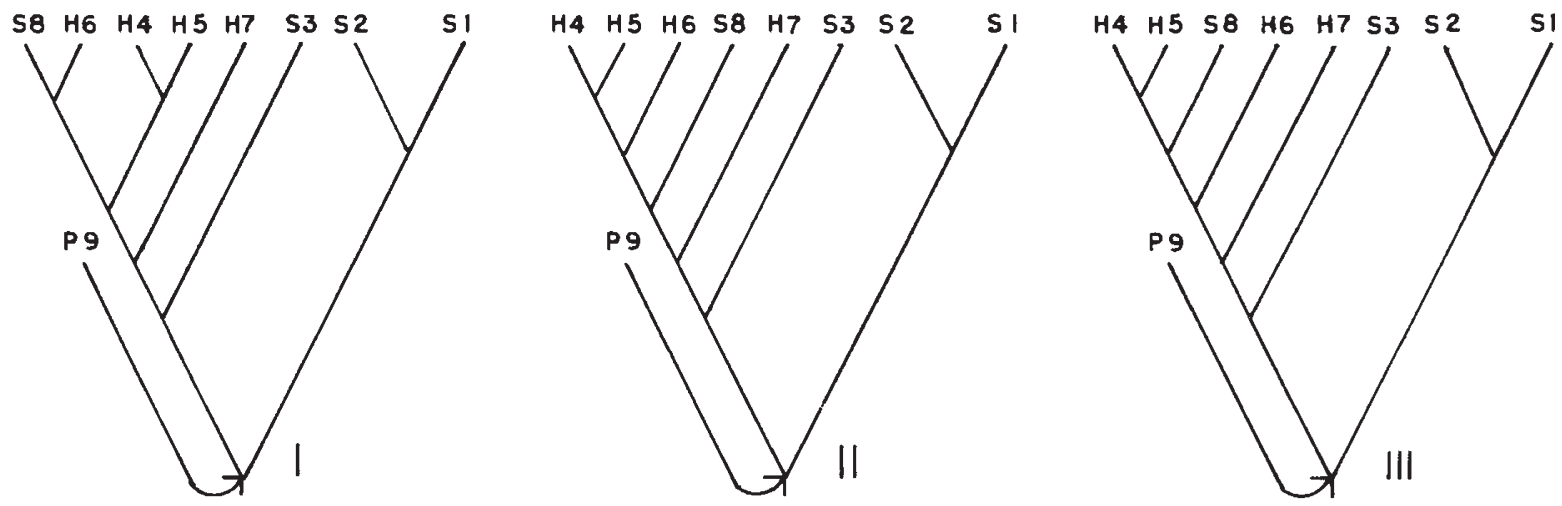

Figure 3 The three maximum parsimony phylogenies generated from the data listed in table 2. The taxa are numbered as follows (S1) D. silvestris Hilo side ancestor, (S2) D. silvestris Maulua (W12B7), (S3) D. silvestris Hualalai ancestor, (H4) D. heteroneura Hualalai (W48G3), (H5) D. heteroneura Hualalai (W48B6), (H6) D. heteroneura Waihaka ancestor, (H7) D. heteroneura Olaa (Q71G12), (S8) D. silvestris Kahuku (U26B9), (P9) D. planitibia Waikamoi, Maui (U84B4).

order that deserves consideration is shown in fig. 5 as Phylogeny IV. This topology coincides with a strict taxonomic branching order and implies a close relationship of all west and east side $D$. silvestris. Because one of the west side $D$. silvestris (U26B9-Kahuku) was shown to be closely related to $D$. heteroneura, possibly as the result of hybridisation (DeSalle et al., 1985), we tested phylogeny V (fig. 5), which represents a branching order based primarily on taxonomy but takes into consideration the problems of hybridisation. Several permutations of Phylogeny $V$ are possible which involve changes in the branching orders of $D$. heteroneura. Phylogeny V represents the topology which fared the best in tests against Phylogeny II. In fact, any topology that involved the branching of the $D$. heteroneura line W48G3 from Hualalai in any position other than the one shown in Phylogeny V may be statistically rejected $(p<0.05)$.
The Hualalai populations are pivotal in the evolution of these two species (Carson, 1983; Kaneshiro and Kurihara, 1982; Kaneshiro, 1983). As the ancestral Hualalai population probably provided the founder of the east side $D$. silvestris (Kaneshiro and Kurihara, 1982), we have tested topologies in which each of the three distinct mtDNA lineages from Hualalai share the most recent common ancestor with the east side $D$. silvestris (Phylogenies V, VI and VII in fig. 6). Finally, we have examined the possibility that because of the recent divergence of these species and the observation that hybridisation between the two species may have occurred, geographic location dictates branching order (Phylogeny VIII, fig. 5).

Table 4 shows the results of applying Templeton's algorithm in the comparison of these various phylogenies. All alternative phylogenies were
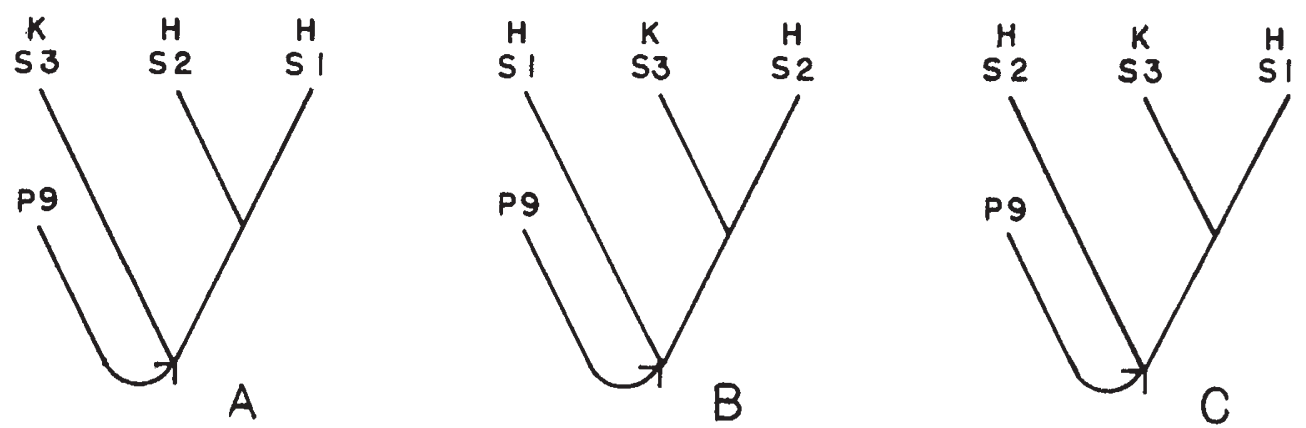

Figure 4 Three possible phylogenetic trees for Hilo side D. silvestris (S1), Maulua D. silvestris (S2) and the Hualalai D. silvestris (S3). D. planitibia (P9) was used as the outgroup for this analysis. H designates east side populations, while $\mathrm{K}$ designates west side populations. 

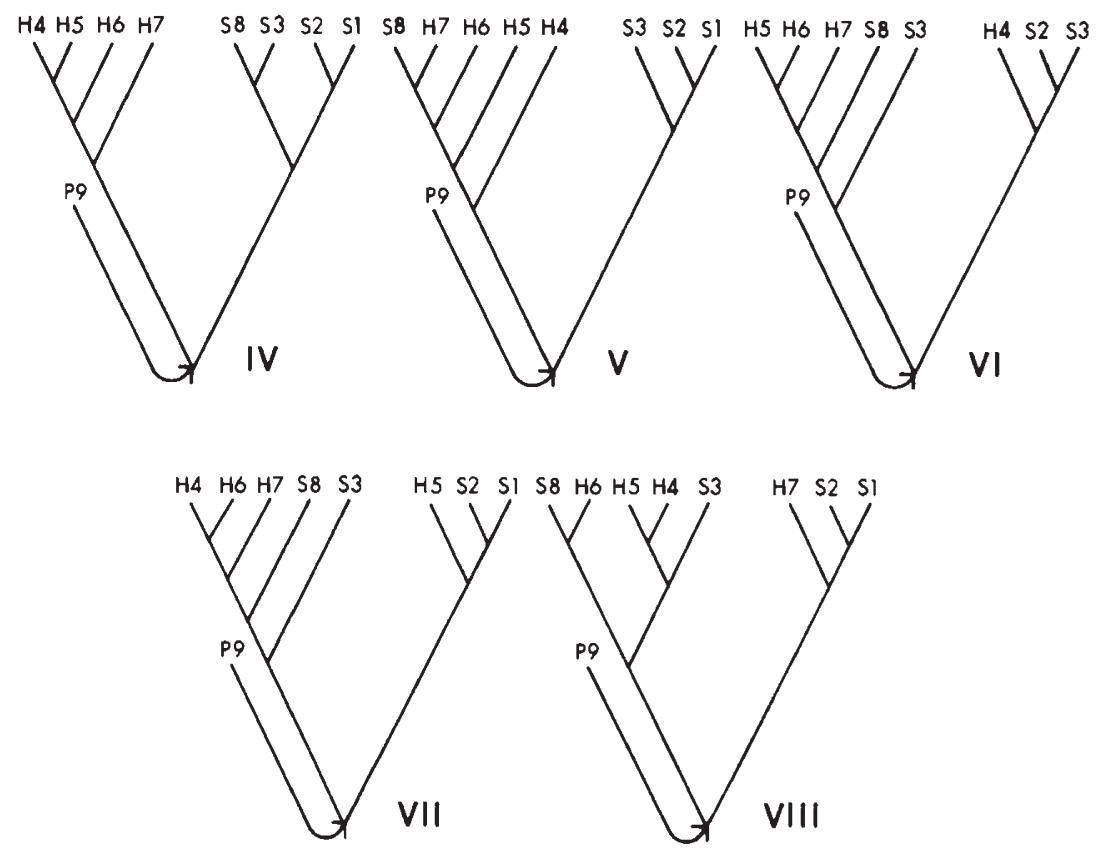

Figure 5 Five additional plausible topologies which were tested against Phylogeny II. See text for rationale for each phylogeny and description of each phylogeny. Taxa are numbered as in fig. 3.

initially compared to Phylogeny II from fig. 3 . When a phylogeny was shown to be as good as this, it was then compared to all the other phylogenies shown in fig. 5. This analysis showed that Phylogeny VI was as probable as Phylogeny II. These two differ only in the placement of one of the $D$. heteroneura from Hualalai (W48G3). All other phylogenies had low probabilities in comparison with Phylogeny II. In agreement with our analysis in fig. 4, table 4 shows that Phylogeny II is seven to eight times more likely than Phylogeny $\mathrm{V}$, the phylogeny utilising classical taxonomy cou- pled with some hybridisation. We have also compared Phylogeny $\mathrm{V}$ to all the alternative phylogenies in fig. 5 (table 4 ) and the results indicate that Phylogeny V is less probable than Phylogenies II and VI.

\section{DISCUSSION}

Chromosome inversions and isozymes are relatively insensitive in reflecting the genetic changes that have occurred during the morphological and

Table 4 Wilcoxon matched pairs signed rank tests for the seven alternative phylogenies given in fig. 5

\begin{tabular}{lccccc}
\hline II vs & IV & V & VI & VII & VIII \\
\hline \# nonzero ranks & 8 & 7 & 8 & 9 & 9 \\
Sum-(ranks) & -3.5 & -7 & -20 & -10 & -10 \\
Probability & $p<0.025$ & $p<0.148$ & $p<0.600$ & $p<0.082$ & $p<0.082$ \\
VI vs & IV & V & VI & VII & VंIII \\
\# nonzero ranks & 8 & 5 & - & 7 & 7 \\
Sum-(ranks) & -7.5 & -4 & - & -6 & -3 \\
Probability & $p<0.080$ & $p<0.250$ & - & $p<0.104$ & $p<0.055$ \\
V vs & IV & V & VI & VII & VIII \\
\# nonzero ranks & 8 & - & - & 8 & 6 \\
Sum-(ranks) & -12 & - & - & -12.5 & -1.5 \\
Probability & $p<0.250$ & - & - & $p<0.275$ & $p<0.039$ \\
\hline
\end{tabular}


behavioural evolution of these populations, but restriction site analysis of mtDNA appears to be very sensitive. This sensitivity is manifest not only in interspecific comparisons, but also in comparisons among populations within a species. An example of this sensitivity is shown in the assessment of the relationships between the east side and west side $D$. silvestris where genetic distance data clearly show that the east side and west side $D$. silvestris have arisen as two discrete maternal lineages (fig. 2). Application of Templeton's algorithm allows the statistical significance of the separation of these two morphological types to be tested (fig. 4). Craddock and Johnson (1979) present isozyme studies which suggest "strong divergence" of west side $D$. silvestris from their east side conspecifics. Sene and Carson (1977), on the other hand, suggest that very little differentiation is shown electrophoretically. The present study on mtDNA includes four restriction sites which show fixed differences between the populations of $D$. silvestris from the two sides of Hawaii.

It appears from mtDNA restriction sites that hybridisation of $D$. silvestris and $D$. heteroneura may have occurred on the west side of Hawaii (DeSalle et al., 1985). In accordance with the mtDNA data, suspected hybrids of these two species have indeed been taken in the field at a west side collecting site (Kaneshiro and Val, 1977). Hybridisation of these two species with subsequent swamping out of the $D$. silvestris mtDNA lineage by the $D$. heteroneura mtDNA lineage might explain the large genetic distance between the east side and west side $D$. silvestris and implies a high degree of similarity between $D$. heteoneura and west side $D$. silvestris at sites where hybridisation is suspected to have occurred. Although certain $D$. silvestris mtDNA lineages are indeed more similar to $D$. heteroneura (W48B2 in fig. 3), most of the west side $D$. silvestris cluster in a cohesive group that is rather distant from sympatric $D$. heteroneura. In fact, the magnitude of the genetic distances between $D$. heteroneura and most west side $D$. silvestris are about the same as between west side and east side $D$. silvestris (table 1). These data suggest that although hybridisation may occur between $D$. heteroneura and $D$. silvestris on the west side, such hybridisation is secondary, and has had little direct impact on the differentiation of the mtDNA lineages and genetic distance measures obtained for restriction sites in mtDNA. We conclude, therefore, that at least three distinct mtDNA lineages exist in these populations, the east side $D$. silvestris, the west side $D$. silvestris, and $D$. heteroneura. This reflects the existence of three distinct morphological lineages among $D$. silvestris and $D$. heteroneura populations.

We have also attempted to infer the phylogenetic relationships of these three lineages. Although we cannot statistically show that a single branching order is superior, we have reduced the possibilities by combining a search for maximum parsimony phylogenies with the application of Templeton's hypothesis testing algorithm. We also compare plausible alternative phylogenies with the best maximum parsimony phylogenies and can reject many of them.

Although table 4 shows that many of the comparisons made with Templeton's hypothesis testing algorithm are not significant at $p<0.05$, the direction of the tests are obvious, implying that Phylogenies II and VI are more probable than all the alternatives. We therefore consider Phylogenies II and VI (figs. 3 and 5, respectively) as the two most likely topologies to explain these data. A common feature of these two superior phylogenies is that one $D$. heteroneura (W48G3) from Hualalai appears to branch in an intermediate position between the three bristle row $D$. silvestris and $D$. heteroneura. The position of this $D$. heteroneura in Phylogeny VI implies morphological convergence of head shape in two separate lineages. However, since it (W48G3) comes from a locality where hybridisation is suspected, it might alternatively be the case that the lineage which gave rise to W48G3 was the product of ancient hybridisation with the two row $D$. silvestris at Hualalai. Phylogeny II is consistent with the morphological data in that no convergence of morphology is required to explain bristle row and head shape in this topology. Whichever of the two phylogenies is superior, one unexpected result emerges, as both imply that the two row $D$. silvestris are closer (VI) or as close (II) to D. heteroneura as they are to the three bristle row $D$. silvestris.

The evolutionary history for present day populations of $D$. heteroneura and $D$. silvestris may therefore reflect their origin from an ancestor or ancestors closely related to $D$. planitibia of Maui and $D$. differens of Molokai (Kaneshiro, 1976). The initial ancestral population was probably established at Hualalai. The position of the Hualalai flies in the mtDNA phylogenies (II and VI) also demonstrates that they are pivotal in the evolutionary history of these flies (Kaneshiro and Kurihara, 1982; Carson, 1983; Kaneshiro, 1983). From this ancestral population at least three distinct mtDNA lineages diverged. One lineage includes the three bristle row $D$. silvestris which spread to the rainforests on the east side of Hawaii. 
On the west side the two remaining lineages, the two bristle row $D$. silvestris and $D$. heteroneura have experienced limited hybridisation, which has had little effect on the overall integrity of the mtDNA lineages. The closer relationship of the west side $D$. silvestris to $D$. heteroneura is also reflected in the ability to obtain viable $\mathrm{F} 1$ from the interspecific crosses (Ahearn et al., 1974; Ahearn and Templeton, 1985). In fact, hybrids of two bristle row or west side $D$. silvestris with $D$. heteroneura appear to be more successful in mating experiments than hybrids between three bristle row $D$. silvestris and west side D. silvestris (Ahearn and Templeton, 1985). This, in conjunction with mtDNA data suggests a recent and perhaps incomplete divergence of these two lineages. The $D$. heteroneura lineage then spread to the east side of Hawaii. We infer that the east side $D$. heteroneura are relatively closely related to the west side $D$. heteroneura by the genetic distance data and Wagner analysis, which show their clustering. This conclusion is also supported by the bristle row data of Carson et al. (1982).

Acknowledgements We thank Alan Templeton for advice on the use of his hypothesis testing algorithm. We also thank $\mathrm{H}$. L. Carson, A. R. Templeton, T. Crease, E. A. Zimmer, C. Simon and $\mathrm{R}$. Zink for comments on earlier drafts. In particular, we would also like to acknowledge the extensive comments of an anonymous reviewer. This work was supported by NIH Grant R01 GM31571 to Alan Templeton.

\section{REFERENCES}

AHEARN, J. N. AND TEMPLETON, A. R. 1985. Interspecific hybrids of Drosophila heteroneura and D. silvestris. I. Courtship success. Evolution (in review).

AHEARN, J. N., CARSON, H. L., DOBZHANSKY, T. AND KANESHIRO, K. Y. 1974. Ethological isolation among three species of the planitibia subgroup of Hawaiian Drosophila. Proc. Natl. Acad. Sci. USA, 70, 901-903.

CARSON, H. L. 1983. Evolution of Drosophila on the newer Hawaiian volcanoes. Heredity, 48, 3-25.

CARSON, H. L. AND BRYANT, P. J. 1979. Genetic variation in Hawaiian Drosophila. VI. Change in a secondary sexual characteristic as evidence of incipient speciation in Drosophila. Proc. Natl. Acad. Sci. USA, 76, 1929-1932.

CARSON, H. L. AND KANESHIRO, K. Y. 1976. Drosophila of Hawaii: systematics and ecological genetics. Ann. Rev. Ecol. Systematics, 7, 311-346.

CARSON, H. L. AND LANDE, R. 1983. Inheritance of a secondary sexual characteristic in Drosophila silvestris. Genetics, 104, s12.

CARSON, H. L. AND TERAMoto, L. T. 1984. Artificial selection for a secondary sexual character in males of Drosophila silvestris from Hawaii. Proc. Natl. Acad. Sci. USA, 81, 3915-3917.
CARSON, H. L., VAL, F. C., SIMON, C. M. AND ARCHIE, J. W. 1982. Morphometric evidence for incipient speciation in Drosophila silvestris from the island of Hawaii. Evolution, 367, 132-140.

CARSON, H. L. AND YOON, Y. S. 1982. Genetics and evolution of Hawaiian Drosophila. In The Genetics and Biology of Drosophila, vol. 13. eds. M. Ashbumer, H. L. Carson and J. N. Thompson, Jr. Academic Press, New York

CRADDOCK, E. M. AND JOH NSON, W. E. 1979. Genetic variation in Hawaiian Drosophila. V. Chromosomal and allozymic diversity in Drosophila silvestris and its homosequential species. Evolution, 33, 137-155.

DESALLE, R. 1984. Mitochondrial DNA evolution and phylogeny in the planitibia subgroup of Hawaiian Drosophila. Ph.D. thesis. Washington University, St. Louis.

DESAlle, R., GIDDings, L. V. AND TEMPLETON, A. R. 1985. Mitochondrial DNA variability in natural populations of Hawaiian Drosophila. I. Methods and levels of variability in $D$. silvestris and $D$. heteroneura populations.

ENGELS, W. J. 1981. Estimating genetic divergence and genetic variability with restriction endonucleases. Proc. Natl. Acad. Sci. USA, 78, 6329-6333.

EWENS, w. J. 1983. The role of models in the analysis of molecular genetic data with particular reference to restriction fragment data. In Statistical Analysis of DNA Sequence Data, ed. by B. Weir. pp. 151-179. Marcel Dekker, New York.

EWENS, W. J., SPIELMAN, R. S. AND HARRIS, H. 1891. Estimation of genetic variation at the DNA level from restriction endonuclease data. Proc. Natl. Acad. Sci. USA, 78, 37483750 .

GIDDINGS, L. V. AND TEMPLETON, A. R. 1983. Behavioral phylogenies and the direction of evolution. Science, 220, 372-378.

HUDSON, R. R. 1982. Estimating genetic variability with restriction endonucleases. Genetics, 100, 711-719.

HUNT, J. A. AND CARSON, H. L. 1982. Evolutionary relationships of four species of Hawaiian Drosophila as measured by DNA reassociation. Genetics, 104, 353-364.

JOHNSON, W. E., CARSON, H. L., KANESHIRO, K. Y., STEINER, W. W. M. AND COOPER, M. M. 1975. Genetic variation in Hawaiian Drosophila. II. Allozymic differentiation in the D. planitibia subgroup. From Isozymes IV: Genetics and Evolution. Ed. C. L. Markert, pp. 563-583. Academic Press, New York.

KANESHIRO, K. Y. 1976. Ethological isolation and phylogeny in the planitibia subgroup of Hawaiian Drosophila. Evolution, 30, 740-45.

KANESHIRO, K. Y. 1980. Sexual selection, speciation and the direction of evolution. Evolution, 34, 437-444.

KANESHIRO, K. Y. 1983. Sexual selection and direction of evolution in the biosystematics of Hawaiian Drosophilidae. Ann. Rev. Entomol., 28, 161-178.

KANESHIRO, K. Y. AND KURIHARA, J. S. 1982. Sequential differentiation of sexual behavior in populations of Drosophila silvestris. Pacific Science, 35, 177-183.

KANESHIRO, K. Y. AND VAL, F. C. 1977. Natural hybridization between a sympatric pair of Hawaiian Drosophila. Amer. Nat., 111, 897-902.

NEI, M. AND LI, W. H. 1979. Mathematical model for studying variation in terms of restriction endonucleases. Proc. Natl. Acad. Sci. USA, 76, 5269-5273.

SENE, F. M. AND CARSON, H. L. 1977. Genetic variation in Hawaiian Drosophila. IV. Close allozymic similarity between $D$. silvestris and $D$. heteroneura from the island of Hawaii. Genetics, 86, 187-198. 
SNEATH, P. AND SOKAL, R. 1973. Numerical Taxonomy. W. H. Freeman and Company, San Francisco.

SPIESS, E. B. AND CARSON, H. L. 1981. Evidence for sexual selection in Drosophila silvestris of Hawaii. Proc. Natl. Acad. Sci. USA, 78, 3088-3092.

SPIETH, H. T. 1981. Drosophila heteroneura and Drosophila silvestris: Head shapes, behavior and evolution. Evolution 35, 921-930.

TEMPLETON, A. R. 1977. Analysis of head shape differences between two interfertile species of Hawaiian Drosophila. Evolution, 31, 630-641.

TEMPLETON, A. R. 1980. The theory of speciation via the founder principle. Genetics, 94, 1011-1038.
TEMPLETON, A. R. $1983 a$. Convergent evolution and nonparametric inferences from restriction fragment and DNA sequence data. In Statistical Analysis of DNA Sequence Data. pp. 151-197. ed. by B. Weir. Marcel Dekker, New York.

TEMPleton, A. R. 1983 b. Phylogenetic inference from restriction endonuclease cleavage site maps with particular reference to the evolution of humans and apes. Evolution, 37, 221-244.

VAL, F. C. 1977. Genetic analysis of the morphological differences between two interfertile species of Hawaiian Drosophila. Evolution, 33, 611-629. 